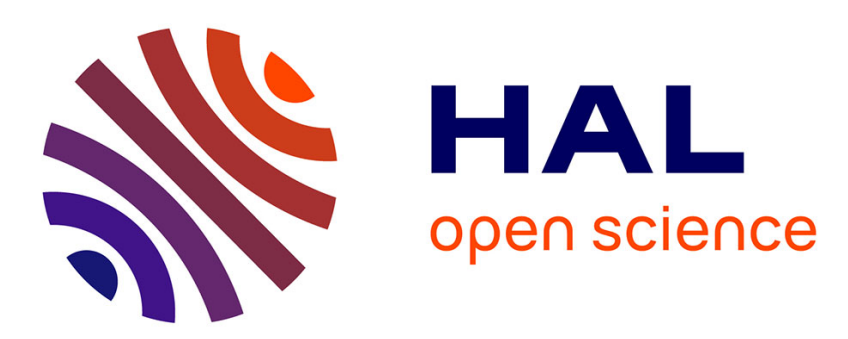

\title{
Study of distributed data fusion using Dempster's rule and cautious operator
}

\author{
Romain Guyard, Véronique Cherfaoui
}

\section{To cite this version:}

Romain Guyard, Véronique Cherfaoui. Study of distributed data fusion using Dempster's rule and cautious operator. 5th International Conference on Belief Functions (BELIEF 2018), Sep 2018, Compiègne, France. pp.95-101, 10.1007/978-3-319-99383-6_13 . hal-01843424v2

\section{HAL Id: hal-01843424 \\ https://hal.science/hal-01843424v2}

Submitted on 17 Oct 2018

HAL is a multi-disciplinary open access archive for the deposit and dissemination of scientific research documents, whether they are published or not. The documents may come from teaching and research institutions in France or abroad, or from public or private research centers.
L'archive ouverte pluridisciplinaire HAL, est destinée au dépôt et à la diffusion de documents scientifiques de niveau recherche, publiés ou non, émanant des établissements d'enseignement et de recherche français ou étrangers, des laboratoires publics ou privés. 


\title{
Study of distributed data fusion using Dempster's rule and cautious operator
}

\author{
Romain Guyard, Véronique Cherfaoui \\ Sorbonne Université, Université de Technologie de Compiègne, CNRS Heudiasyc \\ UMR 7253, France
}

\begin{abstract}
This paper presents new algorithms to process data exchanged in vehicular networks. In previous works, a distributed data fusion method using belief functions to model uncertainties has been proposed for smart cars network. Since the origin of data coming from other cars is unknown, this algorithm uses the idempotent cautious operator in order to prevent data incest. This operator has been proved to be efficient in the case of transient errors and ensures the fusion convergence. However, since the cautious operator is idempotent, the quantity of concordant sources does not change the result of fusion. Thus we propose several schemes adding Dempster's rule in order to improve the fusion when we can ensure that data come from independent sources. We introduce three new combinations layout of Dempster's rule and cautious operator and we compare them using real data coming from experiments involving several communicating cars in the context of the COMOSEF project.
\end{abstract}

\section{Introduction}

Most smart car perception system are based on data coming from embedded sensors. However, despite technological improvements, their capabilities are limited. Thus, we propose to use information coming from other vehicles thanks to wireless network. Two methods are possible. The first one, known as cloud computing, uses a central server that gathers data from all the vehicles, then computes interesting results and sends them back. Even if this method is currently privileged, it has drawbacks. For instance, it introduces latency due to network exchanges that can be a problem in a context of high topological dynamics. Moreover, private data from users are sent to a third party and that can be an invasion of privacy. The other method, studied in this paper consists of peer-to-peer communication between vehicles (VANETs) [1]. By exchanging data with the vehicle neighbors and with neighbors of neighbors it is possible to benefit of other's knowledge to complete our local sensors capacities. Trusting other cars may not always be possible because their data can be wrong, intentionally or not. Thus, the algorithm we will use must work even if some neighbors send false data. Some research shows the possibility of hybrid VANETs and cloud computing [2].

Vehicle networks are different from regular networks because the topology can change at any time. A WIFI norm called 802.11p has been developed for 
this kind of dynamics. In order to converge to a common value, a robust and dynamic algorithm for distributed data fusion has been proposed in [3]. Each node of the network takes its local value and fuses it using the cautious operator [4] with data that neighbors sent to it. Then, it sends the result to all other cars which do the same thing. Thus, a same value is fused at each hop and then we have to be careful to not artificially increase the confidence due to cycle fusion. This algorithm has limitations. Firstly the idempotent cautious operator gives the same result if there is one car or multiple cars with the same local value. Secondly it doesn't fuse data over time. This paper compare alternatives of the fusion process by combining Dempster's rule with the cautious operator in order to increase the importance of the result.

\section{Distributed data fusion algorithms}

\subsection{Belief combination}

Dempster's rule shown in Equation 1 is the most used fusion operator in the belief function framework.

$$
\begin{cases} & \left(m_{1} \oplus m_{2}\right)(\emptyset)=0 \\ A \neq \emptyset & \left(m_{1} \oplus m_{2}\right)(A)=\frac{1}{1-K} \sum_{B \cap C=A} m_{1}(B) m_{2}(C) \\ \text { where } & K=\sum_{B \cap C=\emptyset} m_{1}(B) m_{2}(C)\end{cases}
$$

With Dempster's rule, sources must be independent. In the car to car communication context, independence is not guaranteed, so the fusion operator have to be idempotent. To fulfill this requirement, the cautious operator has been proposed in [4]. This operator is applied on weight using the operator minimum as shown in Equation 2. Let be a variable $\omega$, taking values in a finite set $\Omega$ called frame of discernment, $2^{\Omega}$ the set of subsets of $\Omega$ and $A \subseteq \Omega$ a set. Let $m(A)$ be the mass function and $w(A)$ the weigh function.

$$
\left(w_{1} \otimes w_{2}\right)(A)=\min \left(w_{1}, w_{2}\right)
$$

In order to maximize the truthfulness of the fusion, Dempster's rule should be used in priority if the sources are independent. If they are dependent, the cautious operator must be used.

\subsection{Original algorithm}

This paper is based on the smart cars data fusion algorithm proposed in [3]. Its objective is to detect events using observations originated from multiple cars. The knowledge about an event of the fleet of vehicles converges to a common value (self-stabilization) using car to car communications. Algorithm 1 ( $\mathrm{INv}[\mathrm{u}]$ and OUTv are mass functions), illustrated in Figure 1 ( $\otimes$ represents the cautious operator), shows that the combination is done using the cautious operator. The following section will discuss possible improvement of this operator. Every loop, 
every data represented by masses functions received from neighbor is fused with the local BBA and the result is broadcasted. The $r()$ function is a discounting operation that reduces the importance of old and distant data and has been discussed in [?]. In [5], the authors present a different algorithm that does not need to use the cautious operator. In this method, instead of sending fused data, local data is broadcasted. Since every local data is associated with the source vehicle, there is no data incest, thus Dempster's rule can be used. However, this require a message to be sent for every observation therefore it can be network intensive. Moreover every node knows personal data which can be a privacy issue. Future work should compare the two approaches.

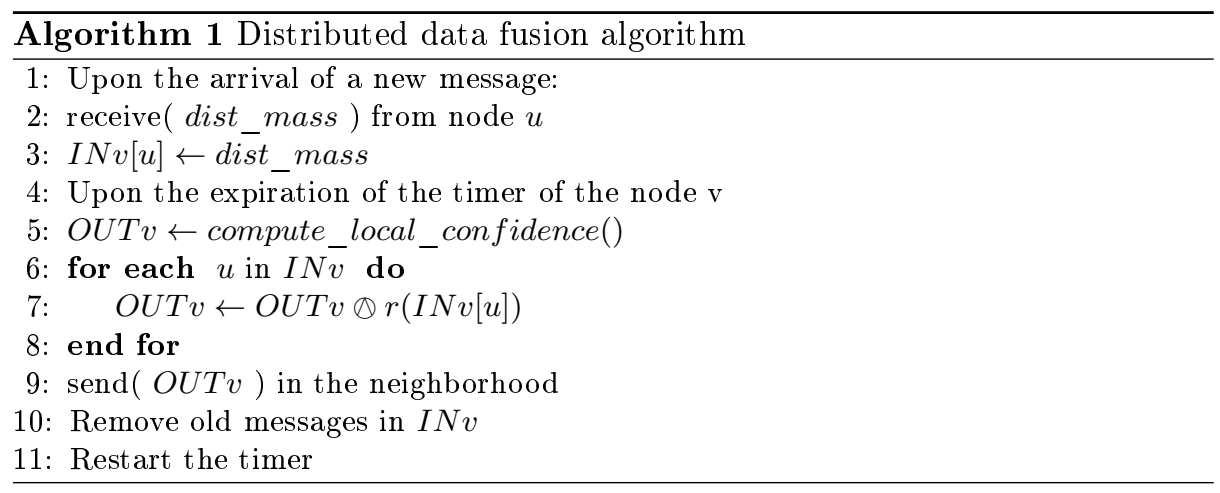

\subsection{Modification of the distributed algorithm}

We propose in this paper to study different scheme of distributed fusion. The idea is to use Dempster's rule that increase the knowledge when data come from two independent sources. The cautious operator should be used otherwise.

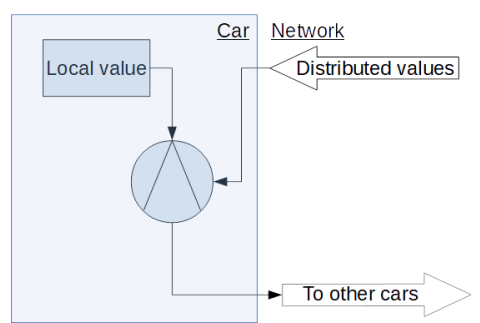

Fig. 1. Cautious operator only

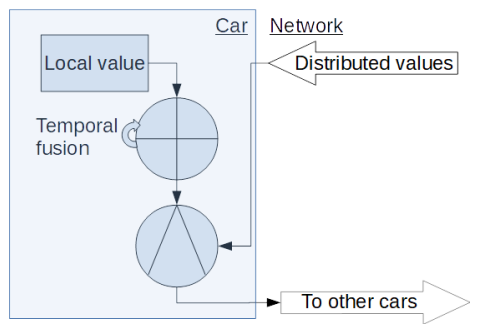

Fig. 2. Dempster's rule before sending 
Indeed, local value is always independent of values coming from the network. It is then possible to combine the with Dempster's rule the local value and the result of the cautious operator applied to all values sent by other cars (distributed values). Figure 2 ( $\oplus$ represents Dempster's rule) shows the fusion diagram of this proposition. As proposed in [6] and commonly done in dynamic data fusion, we can add a temporal fusion of the local value with Dempster's rule. This operation is done before the network data fusion as shown in Figure 3. Finally, we propose a fourth fusion diagram in Figure 4 that assumes that nodes send both distributed and local values. Dempster's rule can be used to combine neighbor local values since independent local data are fused only once.

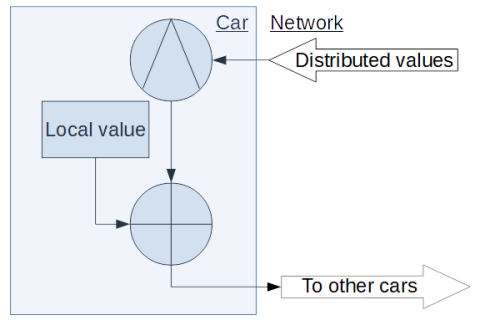

Fig. 3. Local temporal Dempster loop

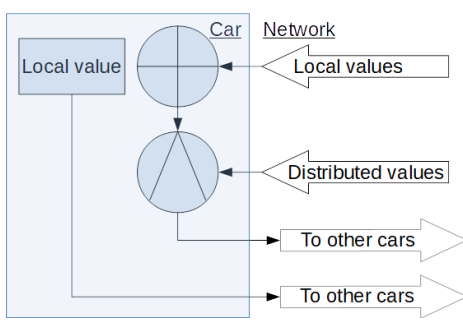

Fig. 4. By sending local values to neighbors

\section{Comparison of fusion scheme with experimental data}

\subsection{Dataset of Comosef project}

The European project CoMoSeF (Cooperative Mobility Service for the Future) has been launched in July 2012 and ended in 2016 [7]. The goal of this project is the creation of services and devices for cooperative application in transports. An experimentation has been done in order to demonstrate the efficiency of the algorithm in the French test site [8]. Heavy rains has been simulated by 10 vehicles in 3 different platoons by using wipers at given positions. The algorithm has generated alerts that has been broadcast to other cars but also to RSU (Road Side Unit). Live alerts generated by the distributed data fusion has been shown on a website.

The original data of the Comosef experimentation has not been recorded. Few days after an equivalent experimentation has been done with 4 cars. There is 4 levels of wiper speed: off, alternate, moderate and fast. Only one car is able to be in alternate mode. In this experimentation, cars follow each other and start their wiper at the same place. The speed increases until the fast mode is reached. The wipers are then stopped without going through intermediate levels. The frame of discernment is $\Omega=\{r, c, s\}$ with $\mathrm{r}$ representing the event "heavy 
rain", $c$ the event "Cloudy" and $s$ the event "Sunny". Masses are computed from the wiper speed using Table 1 . Figure 5 shows for each vehicle the mass on event "heavy rain" with local data coming directly from wipers. The following of this paper will use those data in order to show the importance of how data fusion operators are combined.

Table 1. BBA for Comosef data

\begin{tabular}{|c|c|c|c|c|c|c|c|c|}
\hline Wiper speed & $\emptyset$ & $\{r\}$ & $\{c\}$ & $\{r, c\}$ & $\{s\}$ & $\{r, s\}$ & $\{c, s\}$ & $\Omega$ \\
\hline \hline Off & 0 & 0 & 0 & 0 & 0.8 & 0 & 0 & 0.2 \\
\hline Alternate & 0 & 0.1 & 0 & 0.5 & 0 & 0 & 0 & 0.4 \\
\hline Moderate & 0 & 0.5 & 0 & 0.2 & 0 & 0 & 0 & 0.3 \\
\hline Fast & 0 & 0.8 & 0 & 0 & 0 & 0 & 0 & 0.2 \\
\hline
\end{tabular}

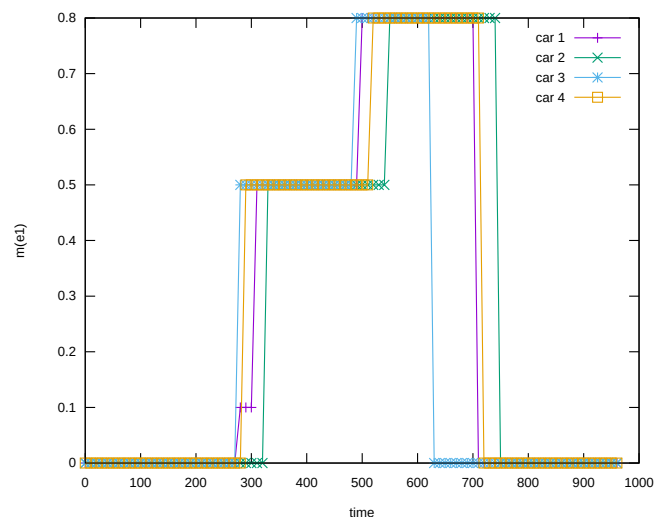

Fig. 5. Local data $m(r)$ of the four cars during the Comosef experimentation

\subsection{Comparison of fusion scheme}

In this section we compare the different combinations of operators using Comosef experimental data.

Comparison in the original scenario Figure 6 shows the result of the fusion using the 4 variants of the algorithm. The top left graph represents the fusion using only the cautious operator. It shows only few changes with the local data. It can be observed that the fusion warns cars of rain before it happens. Even if the same measurement is done multiple time by all the cars, the result of the fusion stays low. The top right and the bottom left graphs show the fusion with 
the Dempster's rule respectively added before and after the cautious operator. They have similar behavior in this case. The Dempster's rule enables the fusion to rise to almost 1 when all cars agree on the same value. The last graph is even closer to reach 1 , even if the wipers are not at full speed.
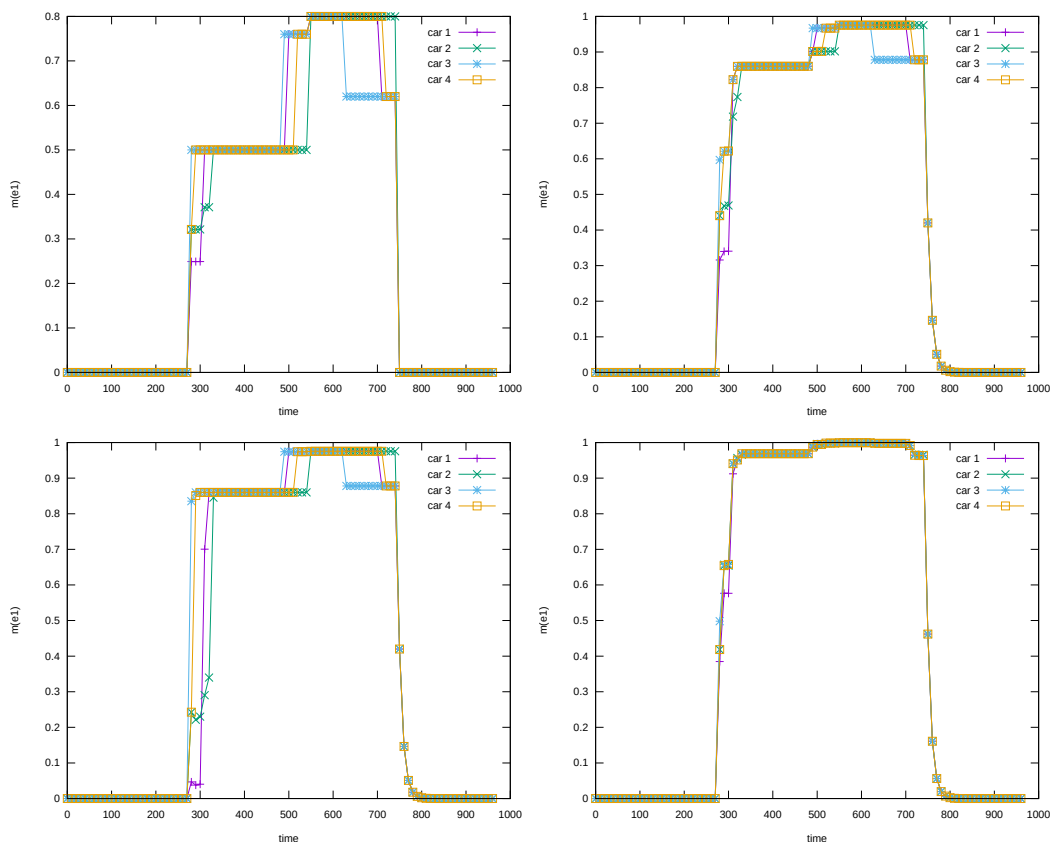

Fig. 6. Comosef data fusion using different combination operators

Comparison with altered scenarios As previously seen, data from the Comosef experiment are almost perfect and every cars communicate without issue. In this part we simulate some changes that could occur in other scenarios. Figure 7 shows the 4 variants of the algorithm supposing that car $\# 1$ has $5 \%$ chance of getting wrong data from the CAN. We can see that the original algorithm is less impacted by transient errors. This behavior comes from the smoothing of Dempster's rule. Errors are only present when cars do not have any information. When the wipers are on, errors are negligible.

In order to compare the fusion on different scenarios, we have created a metric called fusion error. The fusion error is the mean of the differences between the real value and the pignistic probabilities of fused data computed at each time divided by the number of cars. The ground truth is 1 if a wiper is turned on. Table 3.2 shows the fusion errors of the four fusion algorithms in four scenarios. In the original scenario, doing a fusion is better than not doing any fusion. As 

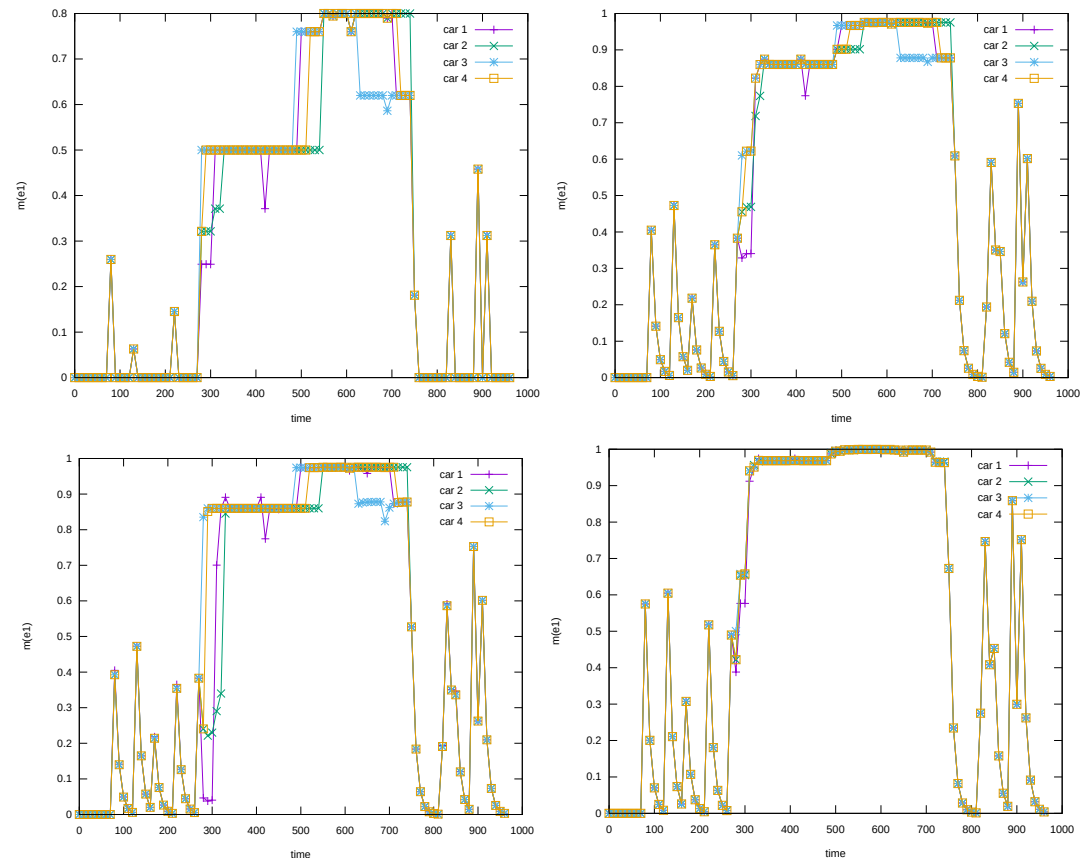

Fig. 7. Comosef data fusion with error injected

previously seen adding Dempster's rule at one hop is the best fusion in this case. In the case of error injected in one car, the difference is lower but still present. When all cars have errors, there is almost no benefit to perform fusion. Finally, we can observe there is no changes in the case the WiFi allows communication only with the vehicles just before and just after the car.

Table 2. Fusion errors: (Fusion $1=$ cautious only, Fusion $2.1=$ with Dempster after cautious, Fusion 2.2 = with local Dempster loop and Fusion $3=$ Fusion with Dempster on neighbor local values)

\begin{tabular}{|c|c|c|c|c|c|}
\hline & No fusion & Fusion 1 & Fusion 2.1 & Fusion 2.2 & Fusion 3 \\
\hline \hline Original scenario & 0.381 & 0.365 & 0.300 & 0.298 & 0.275 \\
\hline Errors car \#1 & 0.383 & 0.374 & 0.326 & 0.325 & 0.309 \\
\hline Errors all cars & 0.387 & 0.395 & 0.368 & 0.375 & 0.364 \\
\hline Low WiFi range & 0.382 & 0.365 & 0.300 & 0.297 & 0.276 \\
\hline
\end{tabular}




\section{Conclusion}

In this paper we have studied different scheme to fuse data in a distributed way. Three algorithms have been presented compared on experimental data. The algorithm given the best result uses Dempster's rule with the data that are guaranteed coming from independent sources and cautious operator else. But this data fusion architecture requires to send more data that could be considerate as private. Future work should focus on testing these algorithms with more cars and in more realistic scenarios.

\section{Acknowledgments}

This work was carried out in the framework of the challenge DAPAD (Distributed and Augmented vehicle Perception to support Autonomous Driving) funded by the Labex MS2T, supported by the French Government, through the program "Investments for the future" managed by the National Agency for Research (Reference ANR-11-IDEX-0004-02). The author would like to thank Bertrand Ducourthial for his work on the Comosef project.

\section{References}

1. F. D. Da Cunha, A. Boukerche, L. Villas, A. C. Viana, and A. A. Loureiro, Data Communication in VANETs: A Survey, Challenges and Applications. PhD thesis, Mar 2014.

2. R. Hussain, J. Son, H. Eun, S. Kim, and H. Oh, "Rethinking vehicular communications: Merging vanet with cloud computing," in Cloud Computing Technology and Science (CloudCom), 2012 IEEE 4th International Conference on, pp. 606-609, IEEE, 2012.

3. B. Ducourthial, V. Cherfaoui, and T. Denoeux, "Self-stabilizing Distributed Data Fusion," Richa A.W., Scheideler C. (eds) Stabilization, Safety, and Security of Distributed Systems. SSS 2012, vol. 7596, pp. 148-162, Oct 2012.

4. T. Denœux, "Conjunctive and disjunctive combination of belief functions induced by nondistinct bodies of evidence," Artificial Intelligence, vol. 172, pp. 234-264, Feb 2008.

5. M. Farah, D. Mercier, F. Delmotte, and É. Lefèvre, "Methods using belief functions to manage imperfect information concerning events on the road in vanets," Transportation Research Part C: Emerging Technologies, vol. 67, pp. 299-320, 2016.

6. N. El Zoghby, Distributed data fusion in VANETs. Phd thesis, Université de Technologie de Compiègne, Feb. 2014.

7. https://www.celticplus.eu/project-comosef/.

8. B. Ducourthial and V. Cherfaoui, "Experiments with Self-stabilizing Distributed Data Fusion," 2016 IEEE 35th Symposium on Reliable Distributed Systems (SRDS), pp. 289-296, Sep 2016. 